Alsina, Angel; Llach, Sílvia (2012). La enseñanza de los sistemas externos de representación matemáticos y lingüísticos en la educación infantil. Revista de Investigación Educativa, 30 (1), 131-144.

\title{
LA ENSEÑANZA DE LOS SISTEMAS EXTERNOS DE REPRESENTACIÓN MATEMÁTICOSY LINGÜÍSTICOS EN LA EDUCACIÓN INFANTIL
}

\author{
Angel Alsina y Silvia Llach \\ Universidad de Girona \\ Instituto de Investigación Educativa
}

\section{RESUMEN}

El objetivo de este estudio es identificar factores que contribuyen a mantener un enfoque básicamente instrumental para enseñar los sistemas externos de representación matemáticos y lingüísticos en las primeras edades de escolarización. En el estudio han participado 123 maestros de Educación Infantil de diversos centros escolares públicos de Andalucía, Asturias, Cataluña y Navarra que han asistido a cursos de formación permanente del profesorado. El análisis cualitativo realizado se basa en grupos de discusión a partir de tópicos. Los resultados evidencian la influencia de diversos factores: el currículo de Educación Infantil; las concepciones del profesorado; las presiones entre etapas educativas; $y$, finalmente, la presión social. La identificación de los factores asociados a una enseñanza instrumental de los sistemas externos de representación puede entenderse como un tipo de información relevante acerca de las condiciones de la enseñanza de estos sistemas que, además, pueden servir de base para reconstruir las prácticas educativas.

Palabras clave: Sistemas externos de representación; Enfoque instrumental; Prácticas educativas; Alfabetización matemática y lingüística.

\footnotetext{
Correspondencia:

Angel Alsina y Sílvia Llach. Universidad de Girona. Instituto de Investigación Educativa. Plaça de sant Domènec, 9. 17071 Girona.

Financiación de la propuesta de artículo:

Este estudio se ha desarrollado en el marco de dos proyectos financiados por organismos públicos: Proyecto I+D+I financiado por el Ministerio de Ciencia e Innovación, 2010-2012 (Ref. EDU2009-13893-C02-02), y Proyecto financiado por el Instituto de Investigación Educativa de la Universidad de Girona, según Resolución del Instituto de Investigación educativa de la Universidad de Girona de 19 de Enero de 2010.
} 


\title{
TEACHING MATHEMATICAL AND LINGUISTIC EXTERNAL SYSTEMS OF REPRESENTATION IN CHILDHOOD EDUCATION
}

\begin{abstract}
The aim of this study is to identify factors that contribute to maintaining an instrumental teaching approach to the teaching of mathematical and linguistic external systems of representation in early school years. The study involved 123 kindergarten teachers from various public schools of Andalucía, Asturias, Cataluña and Navarra (Spain) who had participed in teacher training courses. The qualitative analysis was based on focus groups using topics as a starting point. The analysis of results shows the influence of various factors related to an instrumental approach: influence of early childhood curriculum, teachers' conceptions, pressures between educational levels and social pressure. The identification of factors associated with instrumental teaching of external systems of representation may be regarded as relevant information about the teaching conditions of these systems, and also allows us to reconstruct educational practices.

Keywords: External systems of representation; Instrumental approach, Educational practices, Mathematics and linguistics literacy.
\end{abstract}

\section{INTRODUCCIÓN}

En la etapa de Educación Infantil existe una práctica docente muy afincada que consiste en acceder a los sistemas externos de representación — sobre todo números y letras escritos - sin asociarlos a su comprensión, es decir, se tiende a enseñar de forma mecánica dichos sistemas sin considerar otros conocimientos y formas de representación preliminares que faciliten su comprensión. Desde esta perspectiva, una de las prácticas más habituales en muchas aulas consiste en que los alumnos resigan, copien o dibujen representaciones semióticas a partir de un modelo dado. Esta actividad todavía tan arraigada proviene de perspectivas que históricamente han atribuido un carácter estrictamente instrumental a los sistemas externos de representación (Tolchinski y Solé, 2009).

Diversos autores (Berdonneau, 2008; Janvier, 1987, Martí, 2003; Tolchinsky y Karmilof-Smith, 1993, entre otros) constatan que esta tradición pedagógica repercute sobre todo de manera negativa en la alfabetización matemática y lingüística en las primeras edades, por diversos motivos:

- En primer lugar, porque se propicia que los alumnos aprendan a escribir los códigos simbólicos sin haber garantizado con antelación su comprensión, cuando en realidad sólo deberían representar simbólicamente aquello que comprenden (Martí, 2003).

- En segundo lugar, porque la representación del código escrito convencional implica habilidad motriz, es decir, los alumnos deben aprender la direccionalidad de los signos, representarlos en espacios limitados, etc. Este tipo de aprendizaje consume mucho tiempo, puesto que en las primeras edades no se tiene todavía la suficiente madurez para realizar este tipo de aprendizajes (Berdonneau, 2008). Desde este punto de vista, esta autora afirma que "la caligrafía de las cifras no es indispensable en educación infantil, y es mejor esperar a la etapa sensible propia de 
cada niño, es decir, el momento en que está realmente maduro para este aprendizaje, que se realizará de forma más rápida, fácil y segura" (pág. 295). El problema, pues, no radica tanto en lo que se hace sino en lo que se deja de hacer al dedicar buena parte del tiempo a copiar, reseguir o dibujar representaciones semióticas.

- Y en tercer lugar, porque algunos alumnos tienden a invertir los signos que escriben. Algunos trabajos realizados desde la neuropsicología han puesto de manifiesto que esta alteración puede presentarse de forma aislada (sólo con números escritos) o en asociación con letras y palabras (Hecaen, Angelerges y Houllier, 1962). A pesar de que las inversiones no pueden asociarse todavía a un trastorno neuropsicológico en estas edades, estos autores afirman que cuando se realiza un aprendizaje de forma errónea antes del momento adecuado, su corrección tiende a ser muy difícil.

El objetivo de este estudio, que se enmarca en un dominio de investigación constituido por los sistemas externos de representación (Martí y Pozo, 2000), es identificar los factores que contribuyen a mantener un enfoque básicamente instrumental para enseñar los sistemas externos de representación matemáticos y lingüísticos. Se parte de la base que el establecimiento de estos factores es un paso necesario para poder ir superando este enfoque en la escuela dado que, tal como señalan Martí y Pozo (2000), los sistemas externos de representación no pueden ser considerados tan sólo como medios transparentes para acceder a las representaciones mentales internas o instrumentos usados como base de los procesos cognitivos, sino que son objetos de conocimiento esenciales en la estructuración de la cognición humana.

El análisis se realiza desde dos disciplinas cuyo conocimiento viene mediado por un sistema semiótico, las matemáticas y la lengua, por lo que en el marco teórico de referencia que sigue a continuación se revisan los procesos de adquisición de ambos códigos. Se incide en la adquisición de los números y las letras escritos al tratarse de dos tipos de códigos que, por su valor cognitivo y cultural, constituyen unos de los aprendizajes más importantes de la educación matemática y lingüística de los primeros años de escolarización.

\section{LA ENSEÑANZA DE LOS SISTEMAS EXTERNOS DE REPRESENTACIÓN MATEMÁTICOSY LINGÜÍSTICOS EN LAS PRIMERAS EDADES}

Martí y Pozo (2000) señalan que uno de los retos más significativos de nuestra cultura es la comprensión y uso de los diferentes sistemas externos de representación, puesto que de ello depende que las personas sepan leer y escribir o realizar operaciones aritméticas, por ejemplo. Sin embargo, los estudios que informan directamente sobre las prácticas educativas formales asociadas a la enseñanza de estos sistemas todavía son escasos, por lo que Scheuer, Sinclair, Merlo de Rivas y Tièche-Christinat (2000) indican que las investigaciones sobre el itinerario de adquisición también pueden resultar de interés educativo. Estas autoras señalan que estos trabajos ofrecen a los educadores un marco que facilita comprender mejor la actividad y la producción notacional de los alumnos y, por lo tanto, favorecer su aprendizaje. Atendiendo a estas razones, a continuación se revisa el itinerario de adquisición de los sistemas externos de representación matemáticos y lingüísticos. 


\section{I. La adquisición de los sistemas externos de representación matemáticos}

Los trabajos que han analizado la adquisición de la notación numérica han estudiado tres tipos de representaciones que interactúan constantemente: la representación verbal (el nombre del número); la representación conceptual (el significado del número); y la representación notacional (el número escrito).

Los modelos mentales (Huttenlocher, Jordan y Levine, 1994; Mix, Huttenlocher y Levine, 2002) establecen que la representación verbal de los números sigue las siguientes fases:

- Pretransición 1: representación inexacta basada en una percepción. Inicialmente, se representan cantidades de manera no verbal e inexacta usando uno o más elementos perceptivos como la superficie, la longitud, la densidad, etc.

- Transición 1: representación no verbal exacta. Pronto se desarrolla la habilidad de representar cantidades de forma exacta, debido a tres factores: en primer lugar, la evolución de la individualización de los objetos — que se perciben ya como cantidades discretas, permanentes y diferentes - permite establecer las bases para comprender las correspondencias término a término, identificar y representar colecciones $y$, en consecuencia, comprender de manera informal la equivalencia numérica y los números; en segundo lugar, entre los dos años y medio y los tres años y medio se empieza a desarrollar la habilidad de representar simbólicamente; y, en tercer lugar; otro factor a considerar es la clasificación.

- Transición 2: representación número-palabra. El nivel anterior, junto con el desarrollo de la capacidad de contar, permite representaciones verbales exactas. El modelo de la abstracción progresiva (Resnick, 1992, 1994), que se centra sobre todo en la representación conceptual de los números, parte de la base que los conceptos y el razonamiento van de lo concreto (contexto específico) a lo abstracto (generalización). Resnick establece las fases siguientes:

- Razonamiento protocuantitativo (contexto específico, cualitativo): inicialmente los niños razonan sobre cantidades de forma global.

- Razonamiento cuantitativo en un contexto específico: con la adquisición del conteo (y otros procesos para representar colecciones de forma exacta) los niños pueden razonar acerca de cantidades específicas en un contexto significativo.

- Razonamiento numérico: se pueden hacer razonamientos sin un contexto específico.

- Razonamiento abstracto: los niños llegan a reconocer los principios generales que se aplican a cualquier contexto o número.

Respecto a la representación notacional, Martí (2003) señala que hacia los 2-3 años se empiezan a distinguir los sistemas figurativos (dibujos, imágenes) y los sistemas arbitrarios (números y letras), mientras que la diferenciación entre números y letras se da hacia los 3-4 años. Según Martí, el trabajo de identificación y categorización progresiva del sistema numérico escrito permite forjar la idea que los números, además de ser objetos interesantes en sí mismos, pueden servir para representar una realidad de naturaleza numérica. Desde este marco se consideran los siguientes aspectos:

1. El esquema organizador que domina en las primeras etapas del conocimiento de los números escritos es el de la correspondencia término a término: una de 
las tendencias dominantes en los niños más pequeños es anotar tantos signos como objetos hay en la colección (Sinclair, 1991). En este contexto, los signos producidos se refieren exclusivamente a la cantidad; la notación se compone de caracteres discretos, alineados; y en muchas producciones, un mismo signo es repetido varias veces.

2. Un momento importante es la producción de un signo único como representante de la cantidad: se trata de un rasgo inherente al sistema de numeración decimal de difícil comprensión, dado que se usa un solo signo para designar toda una colección. El hecho de que exista un objeto semiótico ya elaborado - los números escritos - que se transmite culturalmente ayuda a esta construcción, y no es hasta que los niños comprenden que un solo signo puede representar una pluralidad cuando empiezan a usar los números escritos.

3. La comprensión del valor cardinal (una única expresión para representar una cantidad) es fundamental, puesto que constituye el punto de partida para que los niños puedan ir adentrándose en la comprensión de las reglas del sistema: valor posicional, etc. (Lerner, Sadovsky y Wolman, 1994).

\subsection{La adquisición de los sistemas externos de representación lingüísticos}

La adquisición de la notación lingüística implica el desarrollo de dos grandes habilidades lingüísticas: leer y escribir.

En los modelos tradicionales, aprender a leer y escribir consistía en la instrucción directa del código simbólico lingüístico para potenciar estas dos habilidades, y se solía dar en los primeros años de Educación Primaria. Actualmente hay una visión más global del desarrollo de estas habilidades, ya que se considera el contacto que los niños mantienen con un entorno rico en letra impresa y su interacción con él forma parte de la llamada alfabetización temprana (Arnáiz, Castejón y Ruiz, 2002; Teale y Sulzbi, 1986).

Otro aspecto a considerar es la percepción de las unidades lingüísticas desde el punto de vista fonológico. Los símbolos lingüísticos corresponden a sonidos (fonemas), y esta unidad no se reconoce de forma espontánea, no es una unidad integral de la representación del habla, aunque se convierte en una unidad representacional a partir de la experiencia del lenguaje hablado. Por ello, la sensibilidad fonológica promueve el desarrollo de las habilidades de descodificación, porque la correspondencia entre sonidos y símbolos se da a nivel de fonemas (Whitehurst y Lonigan, 2001).

En relación a esta progresión de percepción que es más fonológica, se produce también una progresión en el reconocimiento de las palabras impresas. En este sentido, ha tenido especial relevancia la propuesta de Ehri (1995):

1. Fase prealfabética: se trata de una fase más o menos logográfica, donde se usan indicios visuales para reconocer algunas letras (por ejemplo, la parte inferior de la letra $g$ en gato).

2. Fase alfabética parcial: en esta fase se activa el conocimiento sobre inicios y finales de palabras. Por ejemplo, se puede leer el nombre propio (Sílvia), pero es probable que una palabra como Sandra, con la que comparte el inicio y el final, también sea leída como Sílvia. Las posiciones se controlan por este orden: inicial de palabra, final de palabra y medial de palabra. Desde el punto de vista del 
tipo de fonema, controlan primero las letras y fonemas consonánticos y luego las letras y sonidos vocálicos.

3. Fase alfabética plena: en esta fase se da de forma casi completa la correspondencia entre fonemas y grafías. Además, en esta etapa los niños adquieren habilidad para segmentar y mezclar sonidos.

4. Fase alfabética consolidada: en esta fase se reconocen las palabras rápida y automáticamente, entre otros factores porque se adquiere conciencia de otros niveles gramaticales en la palabra. Por ejemplo, se reconocen tipos de palabras por su estructura morfológica. En estas etapas de consolidación, Ehri propone que las palabras escritas se convierten en mapas fonéticos que distribuyen visualmente los elementos de pronunciación. Así, las conexiones grafofonémicas se erigen como un poderoso sistema mnemotécnico que vincula las palabras escritas, sus pronuncias y sus significados. De esta forma, una vez está consolidado el trazado alfabético, las palabras pueden ser leídas a primera vista.

Si consideramos también las capacidades productivas, vemos que hay una estrecha relación entre la progresión de las unidades de percepción con las primeras representaciones escritas, tal como se establece en Ferreiro y Teberosky (1979):

1. Estadio presilábico: se diferencian las letras de otro tipo de representaciones gráficas.

2. Estadio silábico: la representación de una letra corresponde a una sílaba.

3. Estadio silábico-alfabético: fase de transición donde conviven la estrategia anterior con la siguiente.

4. Estadio alfabético: una letra corresponde a un fonema.

\section{METODOLOGÍA}

\section{I. Muestra}

En el estudio han participado 123 maestros y maestras de Educación Infantil de diversos centros escolares públicos de Andalucía, Asturias, Cataluña y Navarra que asistieron a un curso de formación permanente sobre Didáctica de las Matemáticas o de la Lengua en Educación Infantil, impartido por los investigadores de este estudio durante el curso 2009-2010. En todos los casos, el formato de la actividad de formación fue el mismo: seis sesiones distribuidas en el calendario académico de la forma siguiente: las sesiones 1 y 2 durante el primer trimestre; las sesiones 3 y 4 en el segundo trimestre; y las sesiones 5 y 6 durante el tercer trimestre. La edad del profesorado oscilaba entre los 61 años y los 22 años, con una media de edad de 35,8 años. Del profesorado encuestado, 116 eran mujeres y 7 hombres.

\subsection{Diseño y procedimiento}

Se utiliza una metodología cualitativa a partir de grupos de discusión. Estos grupos se nutren de la discutibilidad que generan las situaciones grupales para obtener información (Elejabarrieta, 1997). Permite que los participantes interactúen, se den apoyo y generen datos que no saldrían sin la estimulación o la participación de otras personas 
en las mismas circunstancias. Normalmente los grupos son de 4-6 personas, pero éstos pueden ir de 3 a 14 participantes (Bloor, Frankland, Thomas y Robson, 2001). Los criterios de inclusión son homogéneos para todos los participantes. Para la obtención de los datos, los formadores usan el listado de tópicos que se expone en la Tabla 1.

TABLA 1

TÓPICOS ALREDEDOR DE LOS CUALES SE GENERAN LOS GRUPOS DE DISCUSIÓN

¿Qué entienden por sistemas externos de representación matemáticos y lingüísticos? ¿Qué piensan que necesitan los alumnos de las primeras edades para aprender los sistemas externos de representación?

¿Qué dificultades tienen para enseñar los sistemas externos de representación?

El procedimiento seguido tiene en cuenta las directrices de Hershkowitz y Schwarz (1999): los maestros se distribuyen en pequeños grupos y debaten durante un tiempo limitado, no superior a 15 minutos, las respuestas. Después del debate, cada grupo escribe los acuerdos y se hace la puesta en común para fomentar un diálogo reflexivo (un portavoz de cada grupo expone las respuestas). Para no crear un sesgo de contenido, en ningún momento los investigadores usan términos calificativos que valoren positivamente y/o negativamente sus respuestas. Los grupos se graban con audio y posteriormente se realiza la trascripción.

Para analizar los datos, a partir de las grabaciones se transcribe tanto la comunicación verbal como la no verbal. El análisis empieza con la lectura de las transcripciones hasta que su contenido nos sea familiar para poder hacernos una primera impresión sobre los datos y en función del objetivo definido ir organizando y estructurando la información. Dicho de otra manera, se empiezan a transformar los "datos brutos" (material original) a "datos útiles".

El análisis categorial se realiza a través de la fragmentación de los textos analizados a partir de la creación de unidades de registro, segmentos de significación. Estas primeras agrupaciones llamadas códigos, son las que a nuestro parecer resumen mejor la información más relevante y significativa para nuestro objetivo. En una segunda fase, renombramos los códigos creados a partir de la utilización del "método de comparaciones constantes" descrito por Strauss y Corbin (1991), que incluye compar'aciones realizadas entre las similitudes, diferencias y conexiones de los datos. Los códigos capturan y condensan significados y acciones, por eso, a medida que se van creando relaciones, comparando códigos, forjando un análisis preliminar de nuestras ideas, los nombres y contenido de los códigos van cambiando, mostrando nuevas relaciones y posibles interpretaciones. A partir de la primera categorización de los datos obtenidos, renombramos, eliminamos, relacionamos, etc. códigos y nos centramos en descubrir los factores que influyen en la metodología de enseñanza de los sistemas externos de representación matemáticos y lingüísticos. Después de este proceso agrupamos los códigos obtenidos en cuatro grandes categorías: la influencia de los currículos de Educación Infantil; las concepciones del profesorado; las presiones entre etapas educativas; y, finalmente, la presión social. 


\section{RESULTADOS}

\section{I. La influencia de los currículos de Educación Infantil}

Algunos de los códigos extraídos de las respuestas sugieren que los currículos derivados de la Ley General de Educación (LGE) de 1970, la Ley de Ordenación General del Sistema Educativo (LOGSE) de 1990 y la Ley Orgánica de Educación (LOE) de 2006, que son los documentos normativos que han regulado el ejercicio profesional del profesorado que actualmente ejerce la docencia en las primeras edades de escolarización, podrían haber incidido en una enseñanza instrumental de los sistemas externos de representación. En la Tabla 2 se presentan algunas transcripciones que señalan en esta dirección.

TABLA 2

LA INFLUENCIA DE LOS CURRÍCULOS DE EDUCACIÓN INFANTIL

"El currículum de mi época decía que los niños debían terminar la etapa de Educación Infantil sabiendo leer y escribir"

"Yo conozco más el currículum de la LOGSE que el actual, pero allí quedaba muy claro que debíamos enseñar a los niños a escribir números y letras"

"El actual currículum exige que los niños se inicien en el conocimiento del código escrito"

Las respuestas de la Tabla 2 sugieren que uno de los factores que determinan las prácticas educativas que los maestros realizan en sus aulas respecto a la enseñanza de los sistemas externos de representación matemáticos y lingüísticos son las orientaciones curriculares. En todos los casos se destaca que es preciso enseñar a los alumnos dichos sistemas durante la etapa de Educación Infantil, aunque con matizaciones importantes: en algunos casos se habla de consolidación de los sistemas, mientras que en otros casos se refieren a la iniciación. En este punto se hace necesario destacar, como se desprende de las dos primeras respuestas, que no siempre esta intervención educativa se ajusta a las directrices actuales, sino que algunas de las maestras entrevistadas tienen como marco de referencia currículos que ya no son vigentes.

Para poder contextualizar y contrastar las respuestas obtenidas, se han analizado las directrices curriculares establecidas en la LGE de 1970, la LOGSE de 1990 y la LOE de 2006. En la LGE las referencias a la Educación Preescolar -que es como se denominó la etapa de Educación Infantil- fueron escasas, y las orientaciones sobre los sistemas externos de representación inexistentes. Este déficit debe interpretarse dentro de un contexto y una época determinados: en su conjunto, la LGE pretendió renovar el marco legal centenario establecido por la Ley Moyano, con un sistema educativo pensado para una sociedad estática, preindustrial y con un nivel de analfabetismo muy elevado.

En el marco de la LOGSE, el Real Decreto 1330/1991, de 6 de Septiembre, por el que se establecen los aspectos básicos del currículo de la Educación Infantil, se proponen nueve Objetivos Generales de la etapa de Educación Infantil, uno de los cuales se 
refiere de forma explícita a la representación: "Representar y evocar aspectos diversos de la realidad vividos, conocidos o imaginados y expresarlos mediante las posibilidades simbólicas que ofrecen el juego y otras formas de representación y expresión" (pág. 29620).

Al realizar un análisis más pormenorizado del Real Decreto se detectan orientaciones más específicas sobre la representación de los códigos simbólicos matemáticos y lingüísticos: "El acceso a los códigos convencionales, que como criterio general debe realizarse en el primer ciclo de la educación primaria, es un largo proceso en el que las posibilidades evolutivas del niño y la intervención pedagógica del educador han de estar en relación para un tratamiento educativo adecuado. La iniciación a los códigos de la lectura y escritura cobra un valor distinto al que se le ha atribuido tradicionalmente, ya que deja de ser el eje alrededor del cual giran las actividades de enseñanza/aprendizaje, convirtiéndose en una meta supeditada a otras ahora más importantes: la motivación por adquirir los nuevos códigos, el acceso a sus características diferenciales, la comprensión y valoración de su utilidad funcional, etcétera". (pág. 29622). Como puede apreciarse, en el currículo se hace referencia a la flexibilidad sobre el momento adecuado de adquirir los sistemas externos de representación. En relación a la lectura y la escritura, la LOGSE supone un cambio de concepción importante, puesto que el aprendizaje del código deja de ser el objetivo y aparecen nuevos factores entre los que se destaca la importancia del uso del código para fines más comunicativos: "La enseñanza sistemática de la lengua escrita no constituye un objetivo de la educación infantil, pero esto no debe impedir el tratamiento de ese sistema, ni la respuesta a los interrogantes que sin duda plantearán los niños, siempre desde un enfoque significativo" (pág. 29622). Del mismo modo que en los parágrafos del código de lectoescritura se detecta un giro importante, también es así respecto a la representación matemática. Como puede apreciarse en el texto que sigue a continuación, se propone que las actividades prácticas y manipulativas sean la base para acceder a este tipo de representación: “En lo que se refiere a la forma de representación matemática, hay que tener en cuenta que el origen del conocimiento lógico-matemático está en la actuación del niño con los objetos y, más concretamente, en las relaciones que a partir de esta actividad establece entre ellos (...). Por esto, la aproximación a los contenidos de la forma de representación matemática debe basarse en esta etapa en un enfoque que conceda prioridad a la actividad práctica" (pág. 29622).

Finalmente, en la Orden ECI/3960/2007, de 19 de diciembre, por la que se establece el currículo y regula la ordenación de la educación infantil, surgida de la LOE de 2006, se explicitan los siguientes aspectos respecto a los sistemas externos de representación: "Iniciarse en los usos sociales de la lectura y la escritura explorando su funcionamiento y valorándolas como instrumento de comunicación, información y disfrute" (pág. 1028). De ello se desprende que las directrices actuales abogan por iniciar la enseñanza de la lectura y la escritura de las notaciones convencionales (números y letras) para ser usadas como instrumentos que ayuden a los alumnos a interpretar y desenvolverse mejor en su entorno. Otro fragmento de dicha Orden destaca que es necesario que los alumnos aprendan a distinguir los diferentes sistemas de representación: "Diferenciación entre las formas escritas y otras formas de expresión gráfica. Identificación de palabras y frases escritas muy significativas y usuales. Percepción de diferencias y semejanzas entre ellas. Iniciación al conocimiento del código escrito a través de esas palabras y frases" (pág. 1029). Como puede apreciarse, estas orientaciones están en sintonía con las aportaciones de Martí (2003), entre otros autores. Sin embargo, al contrastar estas orientaciones curriculares con las 
opiniones del profesorado se observa, en algunos casos, cierto desajuste entre dichas instrucciones y las prácticas de aula. Este desequilibrio se produce, sobre todo, cuando se tienen como marco de referencia currículos que ya no son vigentes en la actualidad, especialmente la LGE de 1970.

\subsection{Presiones entre etapas educativas}

Los documentos normativos analizados en el subapartado anterior establecen una separación entre las etapas de Educación Infantil y Primaria. Obviamente existen motivos de diversa índole para mantener esta separación, pero la existencia del salto entre una y otra etapa genera una dinámica que a veces puede condicionar las prácticas en previsión de etapas futuras. Dicho de otro modo, se puede constatar en algunas respuestas la presión interna que recibe el profesorado de Educación Infantil por parte del profesorado de Educación Primaria, al exigirles que los alumnos accedan a la Educación Primaria sabiendo leer y escribir. Las transcripciones de la Tabla 3 apuntan en esta línea:

TABLA 3

PRESIONES ENTRE ETAPAS EDUCATIVAS

"Los de Primaria nos exigen que cuando los niños lleguen a $1^{\circ}$ sepan leer, escribir palabras y números"

“Cuando la educación de los niños y niñas de 4 a 6 años pasó a llamarse Educación Preescolar, no se consideraba que la lectoescritura se debiera iniciar en estas edades. Esto llevó a que el paso de la Educación Preescolar a la Primaria supusiera un verdadero reto para los profesores de Primaria, ya que los libros de texto venían escritos de tal manera que el niño y la niña tenían que saber leer y escribir".

"Es necesario enseñar a los niños a leer y escribir en Educación Infantil, ya que si no es así los profesores de Primaria pierden mucho tiempo"

En la Tabla 3 se aprecia que para una parte del profesorado encuestado, la etapa de Educación Infantil parece estar sólo al servicio de la etapa de Educación Primaria, dándole sobre todo un trato de educación preescolar, como en anteriores currículos (LGE de 1970) o bien en currículos no implementados, como la Ley Orgánica de Calidad de la Educación (LOCE de 2002). Si bien es cierto que el Sistema Educativo Español está organizado para que las personas vayan adquiriendo en cada etapa educativa conocimientos que los habiliten para cursar las etapas posteriores, no debe olvidarse que cada etapa tiene unas finalidades propias. En el caso de la Educación Infantil, la legislación educativa actual establece ocho objetivos para esta etapa educativa, dos de los cuales inciden en los sistemas externos de representación: " $f$ ) Desarrollar habilidades comunicativas en diferentes lenguajes y formas de expresión; g) Iniciarse en las habilidades lógico-matemáticas, en la lectoescritura y en el movimiento, el gesto y el ritmo" (pág. 1017). Como puede apreciarse, no se explicita que el alumnado deba terminar esta etapa dominando los sistemas, sinó habiéndolos iniciado. 


\title{
4.3. Las concepciones del profesorado
}

Otro factor detectado en las transcripciones analizadas es que parte del profesorado encuestado aún considera que el aprendizaje de los sistemas externos de representación es la principal finalidad en las primeras edades, al pensar que el dominio de estos códigos es imprescindible para poder empezar la etapa de Educación Primaria. En la Tabla 4 se exponen algunos comentarios que ponen de manifiesto esta idea:

\section{TABLA 4}

\section{LAS CONCEPCIONES DEL PROFESORADO}

\begin{abstract}
"Por desgracia, existen profesores que no creen conveniente iniciar al niño en esta tarea (lectura y escritura), ya que consideran que a estas edades no se posee la madurez suficiente. Sin embargo, se observa la conveniencia de iniciar al niño y a la niña en la lecto-escritura".

"Cuando los niños terminan la Educación Infantil deben saber escribir los números del 1 al 9. Por esto en mi clase utilizo cuadernos de escritura de números".

"Para ayudar a los niños a aprender a escribir los letras y los números, uso imágenes de soporte: el 2 con un pato; la letra J con un anzuelo; etc."
\end{abstract}

Las afirmaciones de la Tabla 4 evidencian que las orientaciones curriculares tanto de la LOGSE como de la LOE no se han implementado de forma general, ya sea por desconocimiento y bien por otros motivos, como por ejemplo interpretaciones erróneas, etc. Se detecta también el uso de algunos recursos didácticos discutibles, que pretenden servir de ayuda para el aprendizaje de los códigos matemáticos y lingüísticos. El itinerario que debe seguir el aprendizaje de los códigos simbólicos es entender la naturaleza abstracta de los símbolos y entender también que en el caso de los símbolos matemáticos, se refieren a cantidades o relaciones entre ellas (Zhang y Norman, 1995), mientras que en el caso de los símbolos lingüísticos se relacionan con los sonidos del habla (Tolchinsky y Karmilof-Smith, 1993). Desde esta perspectiva, el hecho de asociar el número dos a la forma de un pato o la letra jota con un anzuelo no facilita el viaje hacia lo simbólico, sino que lo dificulta porque añade un eslabón innecesario, que establece nuevas relaciones de significado entre los sistemas externos de representación y objetos reales parecidos, relaciones que dificultan la comprensión del sistema.

\subsection{La presión social}

Una parte del profesorado que ha participado en el estudio se encuentra en ocasiones con familias que exigen que sus hijos aprendan los sistemas externos de representación a través de los modelos tradicionales con los que ellos aprendieron, basados en la repetición y la práctica. En la Tabla 5 se exponen algunas transcripciones que ponen de manifiesto que, efectivamente, en algunos casos la práctica educativa del profesorado puede verse condicionada por las concepciones de las familias. 
TABLA 5

LA PRESIÓN SOCIAL

\begin{abstract}
"En la reunión de inicio de curso muchas familias me piden que enseñe a leer y a escribir a sus hijos porque ellos a su edad ya lo aprendían, y piden que les de pautas para trabajar en casa este tema".

"En una entrevista una madre me hizo el comentario siguiente: mi hijo ya hace el número uno pero le ha sido muy difícil hacer el dos. Trato de incentivarlo con actividades para que aprenda a hacer el dos pero nada ... tanto que algunas veces no me quiere trabajar y se distrae mucho ¿Cómo puedo hacer para que aprenda a hacerlo?"

"En principio tenía pensado no trabajar los números escritos, pero los padres no estuvieron de acuerdo con esta medida y al final lo estoy trabajando"
\end{abstract}

Estos comentarios dejan entrever que, a menudo, tanto las directrices curriculares — sobre todo de la LOGSE y de la LOE- como los conocimientos aportados por expertos (Berdonneau, 2008, entre otros) sobre el momento adecuado de iniciar la enseñanza de los sistemas externos de representación matemáticos y lingüísticos, entran en contradicción con lo que las familias consideran que deben aprender sus hijos en las aulas de Educación Infantil. Aunque con objetivos colaterales al presente trabajo, Pifarré, Sanuy, Huguet y Vendrell (2003) ya pusieron de manifiesto que la familia, y de forma más concreta variables como la condición lingüística o la situación socio-profesional, pueden influir en el rendimiento académico de los alumnos.

\title{
5. REFLEXIONES FINALES SOBRE LA ENSEÑANZA DE LOS SISTEMAS EXTERNOS DE REPRESENTACIÓN
}

En este artículo se han identificado cuatro factores que pueden explicar el mantenimiento de un enfoque instrumental en la enseñanza de los sistemas externos de representación matemáticos y lingüísticos. Se trata, tal como señalan Tolchinski y Solé (2009), de una visión vinculada al origen histórico de los sistemas externos de representación, que habrían servido como un almacén cultural que permitiría conservar y recuperar la información.

A partir de los datos obtenidos en el estudio, los factores que parecen haber incidido son la interpretación errónea o el desconocimiento de las directrices curriculares actuales; las concepciones de parte del profesorado de Educación Infantil; las presiones entre etapas educativas; y, finalmente, la presión social. La búsqueda de los factores que subyacen en las prácticas de enseñanza y aprendizaje es un paso necesario para superar la enseñanza de los sistemas externos de representación desde esta perspectiva e indagar en un carácter más epistémico, dado que tanto desde el punto de vista cultural como psicológico, muy pronto la función instrumental asociada al mero almacenamiento y recuperación de la información factual va sustituyéndose por un enfoque epistémico que implica funciones cognitivas más complejas. En esta línea, Pérez-Echevarría, Martí y Pozo (2010) señalan que, además de cumplir esta función mnemónica, la información debe ser 
transformada o procesada en nuevos códigos, y estas operaciones de recodificación no sólo deben ser aprendidas sino que se vuelven a su vez instrumentos o funciones cognitivas que permiten nuevas operaciones. Así, por ejemplo, los números que se presentan oralmente deben ser recodificados a un código cuya sintaxis haga posibles operaciones mentales y matemáticas que serían imposibles en este código oral original. Según estos autores, es así como la adquisición y la operación con estos sistemas de representación genera nuevas funciones epistémicas, nuevas formas de conocer y operar sobre mundos simbólicos, no necesariamente presentes. Desde esta perspectiva, el aprendizaje de la numeración y el cálculo no se basa sólo en enseñar a escribir números o los algoritmos de las operaciones; de la misma manera, aprender a escribir no implica sólo enseñar la representación simbólica de las letras, o las reglas ortográficas. En ambos casos, los sistemas externos de representación están al servicio de la representación del mundo. Por ello, la intervención didáctica debe hacer más énfasis en los aspectos relacionales entre los signos y el mundo que en los signos de forma aislada.

En este sentido, la identificación de los factores asociados a una enseñanza instrumental de los sistemas externos de representación puede entenderse como un tipo de información relevante acerca de las condiciones de la enseñanza de estos sistemas. Aún así, será necesario realizar otros estudios en el futuro para analizar diversos aspectos asociados a estas prácticas educativas e indagar en algunas estrategias compartidas entre las dos disciplinas que posibiliten su enseñanza desde un enfoque más epistémico en las aulas de Educación Infantil.

\section{BIBLIOGRAFÍA}

Arnáiz, P., Castejón, L. y Ruiz, M.S. (2002). Influencia de un programa de desarrollo de las habilidades psicolingüísticas en el acceso a la lecto-escritura. Revista de Investigación Educativa, 20(1), 189-208.

Berdonneau, C. (2008). Matemáticas activas (2-6 años). Barcelona: Editorial Graó.

Bloor, M., Frankland, J., Thomas, M. y Robson, K. (2001). Focus groups in social research. Thousand Oaks: Sage Publications.

Ehri, L. C. (1995). Phases of development in learning to read words by sight. Journal of Research in Reading, 18, 116-125.

Eimas, P. D., Siqueland, E. R., Jusczyk, P. W. y Vigorito, J. (1971) Speech perception in early infancy. Science, 171, 304-306.

Elejabarrieta, F. (1997). El método lingüístico. Técnicas de obtención de información. Barcelona: Universitat Autònoma de Barcelona.

Ferreiro, E. y Teberosky, A. (1979). Los sistemas de escritura en el desarrollo del niño. México: Siglo XXI Editores.

Hecaen, H., Angelerges, R. y Houllier, S. (1962). Les varietes cliniques des acalculies au cours des lesions retrorrolandiques: approche statistique du probléme. Revue Neurologique, 105, 85-103.

Hershkowitz, R. y Schwarz, B. (1999). Reflective processes in a mathematics classroom with a rich learning environment. Cognition $\mathcal{E}$ Instruction. 17(1), 65-91.

Huttenlocher, J., Jordan, N. C. y Levine, S. C. (1994). A mental model for early arithmetic. Journal of Experimental Psychology: General, 123, 284-296. 
Janvier, C. (1987). Problems of representation in mathematical learning and problem solving. Hilsdalle NJ: Lawrence Erlbaum Associates.

Lerner, D., Sadovsky, P. y Wolman, S. (1994). El sistema de numeración: un problema didáctico. En P. Parra y I. Sáez (Eds.), Didáctica de las Matemáticas (pp. 95-184). Barcelona: Paidós.

Martí, E. (2003). Representar el mundo externamente. Madrid: Visor.

Martí, E. y Pozo, J. I. (2000). Más allá de las representaciones mentales: la adquisición de los sistemas externos de representación. Infancia y Aprendizaje, 90, 11-30.

Mix, K. S., Huttenlocher, J. \& Levine, S. C. (2002). Math without words: Quantitative development in infancy and early childhood. Nueva York: Oxford University Press.

Pérez-Echevarría, M.P., Martí, E. y Pozo, J.I. (2010). Los sistemas externos de representación como herramientas de la mente. Cultura y Educación, 22(2), 133-147.

Pifarré, M., Sanuy, J., huguet, A. y Vendrell, C. (2003). Rendimiento matemático en contextos bilingües: Análisis de la incidencia de algunas variables del contexto socio-educativo. Revista de Investigación Educativa, 21(1), 183-199.

Resnick, L.B. (1992). From protoquantities to operators: Building mathematical competence on a foundation of everyday knowlodge. En G. Leinhard, R. Putman y R.A. Hattrup (Eds.), Analysis of arithmetic for mathematics teaching (pp. 373-425). Hillsdale, NJ: Erlbaum.

Resnick, L.B. (1994). Situated rationalism: Biological and social preparation for learning. En L.A. HIrschfield y S.A. Gelman (Eds.), Mapping the mind. Domain-specificity in cognition and culture (pp. 474-493). Cambridge: Cambridge University Press.

Scheuer, N., Sinclair, A., de Rivas, S.M. y Tièche-Christinat, C. (2000). Cuando cienta setenta y uno se escribe 10071: niños de 5 a 8 años produciendo números. Infancia y Aprendizaje, 90, 31-50.

Sinclair, A. (1991). Children's production and comprehension of written numerical representations. En K. Durkin y B. Shire (Eds.), Language in mathematical education (pp. 59-68). Buckingham: Open University Press.

Strauss, A. y Corbin, J. (1991). Basics of qualitative research. Grounded theory: procedures and techniques. Newbury Park, CA: Sage Publications.

Teale, W. y Sulzby, E. (1986). Emergent Literacy: Writing and Reading. Norwood, Nueva Jersey: Ablex.

Tolchinsky, L. y Karmilof-smith, A. (1993). Las restricciones del conocimiento notacional. Infancia y Aprendizaje, 62-63, 19-51.

Tolchinsky, L. y Solé, I. (2009). Las condiciones de aprendizaje de la lengua escrita. Infancia y Aprendizaje, 32(2), 131-140.

Whiteturst, G. J. y Lonigan, C. J. (2001). Emergent literacy: Development from Prereaders to Readers. En S.B. Neuman y D.K. Dickinson (Eds.), Handbook of Early Literacy Research (pp. 11-29). Nueva York: The Guilford Press.

Zhang, J. y Norman, D.A. (1995). A representational analysis of numeration systems. Cognition, 57, 271-295.

Fecha de recepción: 12 de enero de 2011.

Fecha de revisión: 05 de febrero de 2011.

Fecha de aceptación: 06 de marzo de 2011. 